\title{
In vivo dosimetry with diodes in rectal cancer patients
}

\author{
Andrej Strojnik \\ Institute of Oncology Ljubljana, Department of Radiophysics, Ljubljana, Slovenia
}

Background. Success of radiotherapy relies on accurate dose delivery. In vivo dosimetry improves control of treatment quality.

Patients and methods. In vivo dosimetry with commercial diodes was performed in 209 rectal cancer patients treated with four-field box technique. The diodes measured either entrance or exit dose in each treatment field. The results were compared to the planned values and the dose delivered to the isocenter was calculated. Tolerance levels were set to 5\% for entrance dose and $8 \%$ for exit dose.

Results. 421 entrance dose and 415 exit dose measurements were performed. The average difference from expected values was $0.9 \%$ for entrance dose (SD 2.1\%) and $-0.5 \%$ for exit dose (SD 3.3\%). In 209 patients, the average absorbed dose in the isocenter differed from the planned values by $0.2 \%$ (SD 1.4\%). Measurement results exceeded the tolerance levels in two patients.

Conclusion. Smaller standard deviation of absorbed dose to the isocenter (1.4\%), compared to those of entrance $(2.1 \%)$ and exit dose measurements (3.3\%), confirms a correlation between the entrance and exit dose deviations of pairs of opposed fields. The fact that during this study in vivo dosimetry exposed two cases of potentially inaccurate treatments proves its necessity.

Key words: rectal neoplasms - radiotherapy; radiotherapy dosage

\section{Introduction}

Effectiveness of radiotherapy greatly depends on the accuracy of the absorbed dose to the tumor and the surrounding tissue. Complementary to portal imaging which helps verifying the position and shape of the treatment fields, in vivo dosimetry provides dosimetric information

Received 9 December 2007

Accepted 22 December 2007

Correspondence to: Andrej Strojnik, Department of Radiophysics, Institute of Oncology Ljubljana, Zaloska cesta 2, SI - 1000 Ljubljana, Slovenia. Phone: +386 15879 631; E-mail: astrojnik@onko-i.si regarding actual treatment delivery. It is understandably considered an indispensable quality assurance procedure and a safety measure in the treatment process. ${ }^{1-3}$ This paper describes the calibration of dosimetric diodes and presents the results of in vivo dosimetry in 209 rectal cancer patients. ${ }^{4}$

\section{Materials and methods}

\section{Calibration}

Two dosimetric diodes EDP-20, manufactured by Scanditronix, were calibrated 
against an ionization chamber in a $15 \mathrm{MV}$ photon beam from a Varian 2100CD linear accelerator. In reference conditions, each diode was taped to a plastic water phantom (dimensions: $20 \mathrm{~cm} \times 30 \mathrm{~cm} \times 30 \mathrm{~cm}$ ) at a distance of $100 \mathrm{~cm}$ from the accelerator focus, in the center of an open treatment field measuring $10 \mathrm{~cm} \times 10 \mathrm{~cm}$ and with gantry angle set to $0^{\circ}$. The ionization chamber was irradiated with the same treatment parameters at depth dose maximum, $2.5 \mathrm{~cm}$ below the phantom surface. In addition to the calibration factor also correction factors accounting for non-reference conditions (different focus surface distances, field sizes, wedged filters and exit dose measurement) were determined. ${ }^{5}$ During each set of measurements all other parameters, apart from the one in question, were kept at reference values. In the case of exit dose correction factor, the gantry was rotated to $180^{\circ}$ and the focus surface distance to the near side of the slab was set to $100 \mathrm{~cm}$. The signal dependence on the gantry angle was investigated up to $20^{\circ}$.

Throughout the calibration and in vivo measurements, each diode was connected to a dedicated channel on an emX Scanditronix electrometer. The electrometer was connected to a computer running DPD12-pc software also provided by Scanditronix Wellhofer. Dark current drift and offset of the assembly were measured and accounted for.

\section{In vivo measurements}

The two diodes were used in routine measurements in rectal cancer patients treated with four-field box technique with the isocenter in the center of the planning target volume. With this technique, the beams delivered the dose to the target from four directions, with the gantry angle values of $270^{\circ}, 0^{\circ}, 90^{\circ}$ and $180^{\circ}$. Such configuration allowed the diode taped to the patient's skin on the $0^{\circ}$ beam's axis to measure not only the $0^{\circ}$ beam's entrance dose, but also the $180^{\circ}$ beam's exit dose. The same principle applied to the $90^{\circ}$ and $270^{\circ}$ beams. The clinical routine for each patient was as follows: the patient was set-up in the correct treatment position as established at the CT simulator and the diodes were taped to the patient's skin at the entrance points of $0^{\circ}$ and $90^{\circ}$ beams. As the treatment started with the accelerator gantry at $270^{\circ}$, the measurement of the $270^{\circ}$ beam's exit dose was first performed, followed by the measurement of the $0^{\circ}$ beam's entrance dose, then of the $90^{\circ}$ beam's entrance dose and concluding with that of the $180^{\circ}$ beam's exit dose. The measurement readings were multiplied by appropriate calibration and correction factors and compared to the values calculated by the planning system. If the difference exceeded the tolerance level of $5 \%$ for entrance dose or $8 \%$ for exit dose, a thorough investigation of treatment parameters was performed together with a scrupulous review of the treatment plan; in vivo dosimetry was repeated at the next treatment session and focus skin distances were carefully measured to verify the correct placement of the dosimeters with respect to the accelerator's focus. If the problem persisted, the treating radiation oncologist was consulted: if portal images of the treated area were satisfactory, the number of monitor units of the problematic treatment field was adapted and another session of in vivo dosimetry was required.

From the deviations of entrance and exit dose measurements from expected values, the deviation of absorbed dose in the isocenter was estimated for each patient. This was accomplished by averaging the deviations of all four fields. The rationale for this was that the beams were weighed equally in the isocenter within a few percent. The proof is as follows: 
Let $D$ be the planned dose in the isocenter and let $w_{i}$ denote the planned weight of the $i$-th beam. Further, let $\varepsilon_{i}$ represent slight perturbations of the beam weighing in the ideal four-field box technique

$$
\begin{aligned}
& D=\sum_{i=1}^{4} w_{i}=1 \\
& w_{i}=\frac{D}{4}\left(1+\varepsilon_{i}\right) \\
& \varepsilon_{i}<<1
\end{aligned}
$$

It follows that

$$
\sum_{i=1}^{4} \varepsilon_{i}=0
$$

For each beam, the deviation of measured and expected entrance (or exit) dose is equal to the deviation of delivered $\left(w_{i}{ }^{\prime}\right)$ and planned $\left(w_{i}\right)$ dose contribution by that beam in the isocenter. Let $\mu_{i}$ be that deviation. Then

$$
\begin{aligned}
& w_{i}{ }^{\prime}=w_{i}\left(1+\mu_{i}\right) \\
& \mu_{i}<<1
\end{aligned}
$$

The actual absorbed dose to the isocenter, $D^{\prime}$, is

$$
D^{\prime}=\sum_{i=1}^{4} w_{i}{ }^{\prime}=\frac{D}{4} \sum_{i=1}^{4}\left(1+\varepsilon_{i}\right)\left(1+\mu_{i}\right)=D\left(1+\frac{1}{4} \sum_{i=1}^{4} \mu_{i}+\frac{1}{4} \sum_{i=1}^{4} \varepsilon_{i} \mu_{i}\right)
$$

Leaving out the last term of the sum as the second order correction, the deviation from the expected dose can be expressed as

$$
\frac{D^{\prime}-D}{D} \approx \frac{1}{4} \sum_{i=1}^{4} \mu_{i}
$$

The fact that the absolute values of $\varepsilon_{i}$ never exceeded $7 \%$ and that the values of $\mu_{i}$ remained within $\pm 5 \%$ for entrance dose and $\pm 8 \%$ for exit dose, which kept the omitted term within $\pm 0.5 \%$, justifies the above approximation.

In all patients, in vivo dosimetry was performed at the second treatment session (portal imaging at the first session). Measurements in the same patient were repeated only in cases of exceeded tolerances due to primary beam attenuation by the diode build-up cap which increases the skin dose and reduces the dose at greater depths. ${ }^{6}$

\section{Results}

\section{Calibration}

Diodes were calibrated for the clinical dose rates of approximately $3 \mathrm{~Gy} / \mathrm{min}$. The signal remained constant throughout the dose rate interval between $1 \mathrm{~Gy} / \mathrm{min}$ and 6 $\mathrm{Gy} / \mathrm{min}$. The diode response linearity was 
tested for the clinical doses between $10 \mathrm{cGy}$ and 10 Gy with the results within the measurement error well below $1 \%$. The diodes were irradiated in geometric conditions which are commonly encountered in the treatment of patients. These include focus skin distances from $75 \mathrm{~cm}$ to $115 \mathrm{~cm}$ with the field sizes between $5 \mathrm{~cm} \times 5 \mathrm{~cm}$ and 30 $\mathrm{cm} \times 30 \mathrm{~cm}$. The diodes were also irradiated with wedged beams, nominal inclinations of hard wedges being $15^{\circ}, 30^{\circ}$ and $45^{\circ}$. Correction factors are presented in Tables 1,2 and 3. Exit dose measurements were performed with the thicknesses of the plastic water phantom ranging from $20 \mathrm{~cm}$ to $35 \mathrm{~cm}$ simulating different patient thicknesses. The exit dose correction factor was found to be almost constant (within 1\%) and was established to be 1.10 and 1.12 for the diodes 1 and 2, respectively. The influence of the gantry angles of up to $20^{\circ}$ was below $1 \%$. Due to the applied four-field box technique and pelvic area topology, the beams were assumed to be perpendicular to the patient's skin surface on which the diode was taped. Gantry angle correction factors were therefore omitted.

Temperature dependence was not investigated. It is practically impossible to monitor the temperature of the detector during the treatment of patients and apply an adequate correction (it takes several minutes for a diode, after being taped on the patient, to reach thermal equilibrium with the $\operatorname{skin}^{7}$ ). According to some guidelines ${ }^{2}$, the influence of temperature may be neglected.

It was estimated that during the time of use the diodes absorbed approximately 200 Gy. No sensitivity degradation was observed.

\section{In vivo measurements}

In vivo dosimetry was conducted in 209 patients. In 6 (3\%) out of 209 patients, in vivo measurements exceeded the tolerances. In 2
Table 1. Focus skin distance correction factors

\begin{tabular}{cc}
\hline $\begin{array}{c}\text { Focus Skin Distance } \\
(\mathbf{c m})\end{array}$ & Correction factor \\
\hline 75 & $0.94 *$ and $0.95 * *$ \\
80 & 0.96 \\
85 & 0.98 \\
90 & 0.99 \\
95 & 0.99 \\
100 & 1 \\
105 & 1.01 \\
110 & 1.01 \\
115 & 1.02 \\
\hline *
\end{tabular}

* Diode 1 and ** Diode 2

Table 2. Field size correction factors

\begin{tabular}{cc}
\hline Field Size $(\mathbf{c m} \times \mathbf{~ c m})$ & Correction factor \\
\hline $5 \times 5-20 \times 20$ & 1 \\
$25 \times 25-30 \times 30$ & 1.01 \\
\hline
\end{tabular}

Table 3. Wedge correction factors

\begin{tabular}{cc}
\hline Wedge $\left(^{\circ}\right)$ & Correction factor \\
\hline 15 & 1.01 \\
30 & 1.02 \\
45 & 1.02 \\
\hline
\end{tabular}

$(33 \%)$ of the 6 even repeated measurement results were beyond acceptable levels. In the first of the above two patients, a closer inspection revealed a false CT image set had been assigned to the patient. A new therapy plan was later created with the correct CT image set. In the second of the above two patients the source of error proved to be a set of incomplete CT images: due to the size of the patient the outmost parts of the patient's hips had not been captured by the CT scanner. To rectify the problem, the focus skin distances in the lateral fields were measured and the number of monitor units was adapted. After the corrections, in vivo dosimetry was repeated and the results were within the tolerance levels in both cases. Excluding 8 treatment sessions with the measurement results outside the accept- 


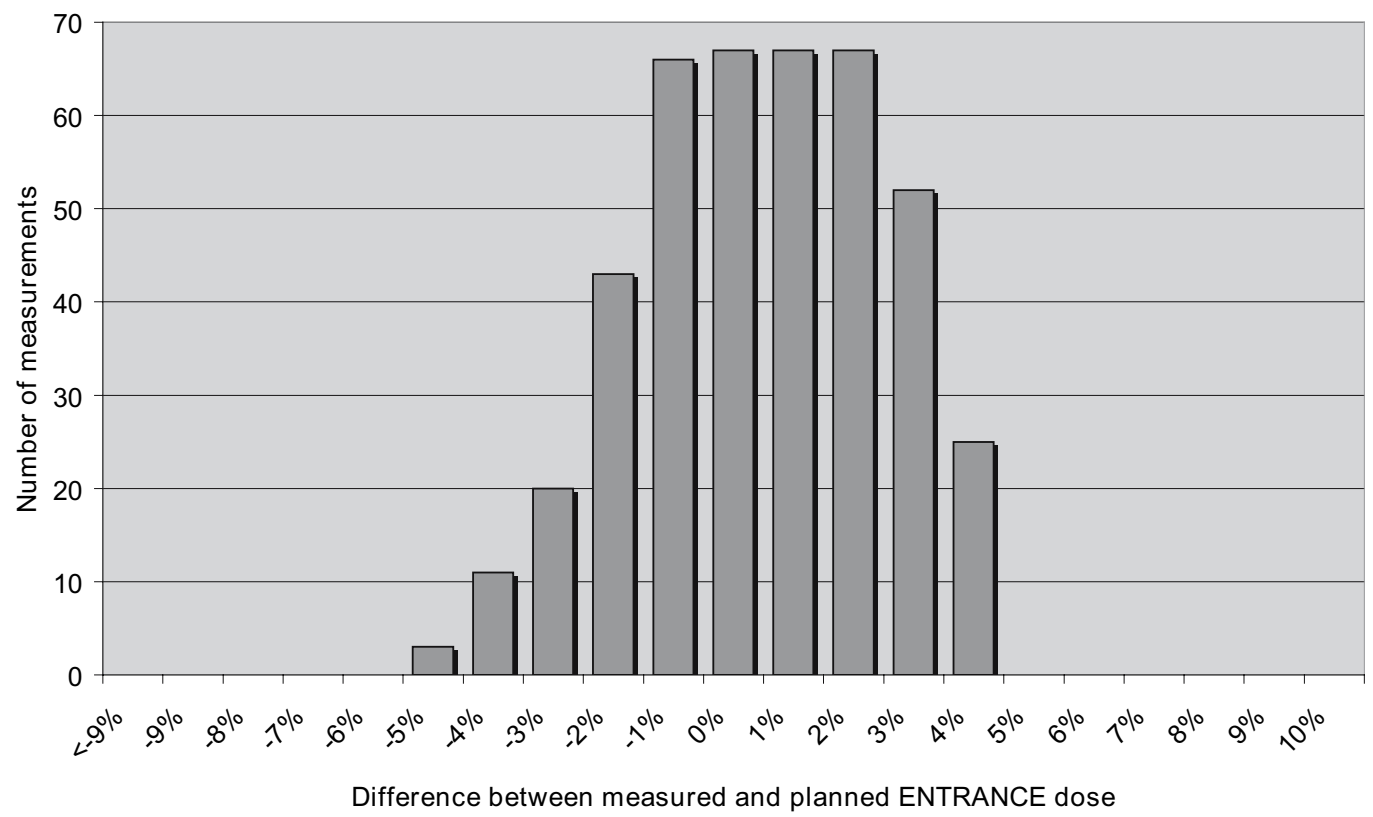

Figure 1. Frequencies of differences between measured and expected entrance doses. In 421 measurements, the average difference was $0.9 \%$ with the standard deviation of $2.1 \%$. The Gaussian distribution is chipped on the right because 6 measurements between $5 \%$ and $8 \%$ were excluded from the chart. These measurements were later repeated and the results were within tolerances.

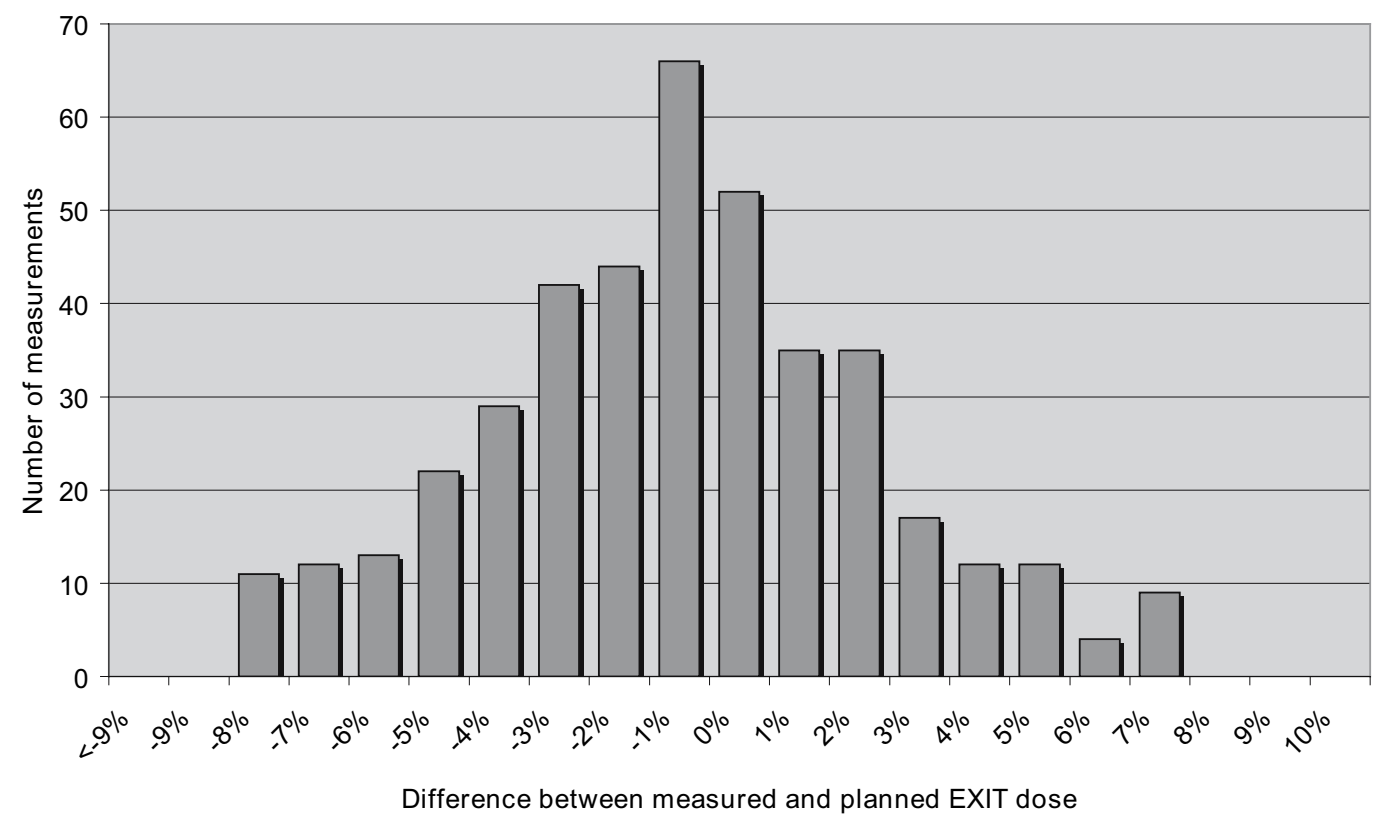

Figure 2. Frequencies of differences between measured and expected exit doses. In 415 measurements, the average difference was $-0.5 \%$ with the standard deviation of $3.3 \%$. 4 values between $-10 \%$ and $-8 \%$ were excluded from the chart. Repeated measurement results were inside the acceptable limits. 


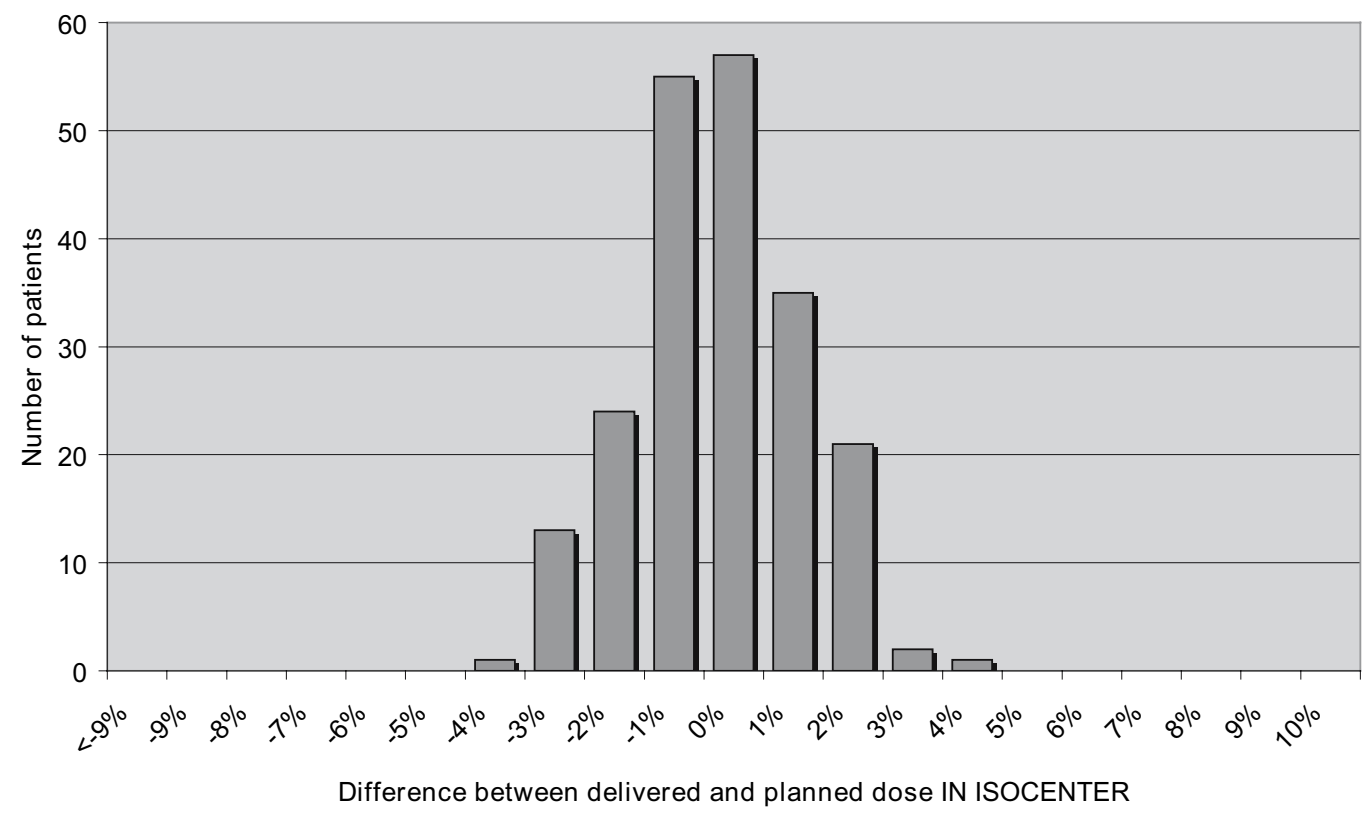

Figure 3. Frequencies of differences between delivered and expected doses in isocenter. In 209 patients, the average difference was $0.2 \%$ with the standard deviation of $1.4 \%$.

able intervals, 421 entrance dose and 415 exit dose measurements were carried out (3 exit dose measurements were overlooked by mistake and replaced by entrance dose measurements in the next treatment session). The average deviation from expected values was $0.9 \%$ for entrance dose (SD 2.1\%) and $-0.5 \%$ for exit dose (SD 3.3\%). In 209 patients, the average absorbed dose in the isocenter differed from the planned values by $0.2 \%$ (SD $1.4 \%$ ). Difference distributions are presented in Figures 1,2 and 3.

\section{Discussion}

The wider spread of exit dose deviations (SD 3.3\%) in comparison to the entrance dose deviations (SD 2.1\%) was suspected to be due to various bowel fillings of patients; however, no further investigation was conducted. The lesser standard deviation of the differences between actual and ex- pected dose in isocenter $(1.4 \%)$ confirmed a correlation between the entrance and exit dose deviations of any pair of opposed beams: if a diode was closer to the accelerator focus than expected when measuring the entrance dose, it was also farther than expected when measuring the exit dose - and vice versa.

In the group of 209 patients, in vivo dosimetry revealed and prevented two cases of inaccurate treatment. In both cases, the cause of error was traced to geometrical inconsistencies related to incorrect CT data used by the planning system. Both problems occurred as a consequence of human errors and no equipment malfunction was discovered. Such discrepancies could have also been detected by the optical distance indicator! Therefore a quick focus skin distance check could prove a valuable quality assurance procedure in the departments that have not yet been outfitted with in vivo dosimetric equipment. 


\section{Acknowledgment}

The study was done partly within a national program Development and Evaluation of New Approaches to Cancer Treatment P30003 financially supported by the Slovenian Research Agency (ARRS).

\section{References}

1. Van Dam J, Marinello G. Methods for in vivo dosimetry in external radiotherapy. ESTRO Booklet No. 1, Leuven: Garant, 1994.

2. Huyskens DP, Bogaerts R, Verstraete J, Lööf M, Nyström H, Fiorino C, et al. Practical guidelines for the implementation of in vivo dosimetry with diodes in external radiotherapy with photon beams (entrance dose). ESTRO Booklet No. 5, Leuven: Garant, 2001.

3. AAPM Report No. 87: Diode in vivo dosimetry for patients receiving external beam radiation therapy. Medical Physics Publishing, 2005.

4. Rikner G, Grusell E. General specifications for silicon semiconductors for use in radiation dosimetry. Phys Med Biol 1987; 32: 1109-17.

5. Leunens G, Van Dam J, Dutreix A, van der Schueren E. Quality assurance in radiotherapy by in vivo dosimetry. 1. Entrance dose measurements, a reliable procedure. Radiother Oncol 1990; 17: 141-51.

6. Nilsson B, Ruden BI, Sorcini B. Characteristics of silicon diodes as patient dosemeters in external radiation therapy. Radiother Oncol 1988; 11: 279-88.

7. Grusell E, Rikner G. Evaluation of temperature effects in p-type silicon detectors. Phys Med Biol 1986; 31: $527-34$. 